\title{
QUALIDADE PÓS-COLHEITA DE MELÃO 'ORANGE FLESH’ MINIMAMENTE PROCESSADO ARMAZENADO SOB REFRIGERAÇÃO E ATMOSFERA MODIFICADA ${ }^{1}$
}

\author{
BRÍGIDA MONTEIRO VILAS BOAS ${ }^{2}$, MÔNICA ELIZABETH TORRES PRADO ${ }^{3}$, EDUARDO VALÉRIO DE BARROS \\ VILAS BOAS ${ }^{4}$, ELISANGELA ELENA NUNES 5 , FRANCISCA MARTA MACHADO CASADO DE ARAÚJO ${ }^{6}$, \\ EDIMILSON BOSCO CHITARRA ${ }^{4}$
}

\begin{abstract}
RESUMO - Melões 'Orange Flesh' minimamente processados foram armazenados por 8 dias sob refrigeração $\left(6 \pm 1^{\circ} \mathrm{Ce} 90 \pm 5 \%\right.$ UR) e atmosfera modificada (passiva e ativas: $5 \% \mathrm{O}_{2}+5 \% \mathrm{CO}_{2}$ e $2 \% \mathrm{O}_{2}+10 \% \mathrm{CO}_{2}$ ). As variáveis $\mathrm{pH}$, acidez titulável, sólidos solúveis e acetaldeído não foram influenciadas pelas atmosferas estudadas. Aumentos na concentração de $\mathrm{CO}_{2}$ ocorreram ao logo do armazenamento, sendo que a atmosfera modificada ativa $\left(2 \% \mathrm{O}_{2}+10 \% \mathrm{CO}_{2}\right)$ foi mais eficaz no controle da produção de $\mathrm{CO}_{2}$ até o quarto dia de armazenamento.

Termos para indexação: Cucumis melo L., vida de prateleira, dióxido de carbono, acetaldeído, etanol.

\section{POSTHARVEST QUALITY OF FRESH-CUT 'ORANGE FLESH’ MELON STORED UNDER REFRIGERATION AND MODIFIED ATMOSPHERE}

\begin{abstract}
Fresh-cut 'Orange Flesh' melons were stored for 8 days under refrigeration $\left(6 \pm 1^{\circ} \mathrm{C}\right.$ e $90 \pm 5 \%$ RH) and modified atmosphere (passive and active: $5 \% \mathrm{O}_{2}+5 \% \mathrm{CO}_{2}$ and $2 \% \mathrm{O}_{2}+10 \% \mathrm{CO}_{2}$ ). The variables $\mathrm{pH}$, titratable acidity, soluble solids and acetaldehyde were not affected, differently, for the atmospheres studied. Increase in $\mathrm{CO}_{2}$ concentration occurred over the storage period. Active modified atmosphere $\left(2 \% \mathrm{O}_{2}+10 \% \mathrm{CO}_{2}\right)$ was the most effective in controlling the $\mathrm{CO}_{2}$ production until the fourth day of storage.
\end{abstract}

Index terms: Cucumis melo L., shelf-life, carbon dioxide, acetaldehyde, ethanol

\section{INTRODUÇÃO}

Dentre as tecnologias disponíveis e em desenvolvimento, o processamento mínimo de frutas surge como uma das principais entre aquelas em ascensão no mercado, pois segue a tendência mundial de consumo de produtos in natura ou mais próximo possível destes. Esta tecnologia permite a obtenção de um produto com características sensoriais e nutricionais praticamente inalteradas e de grande conveniência para o consumo imediato, ou seja, sem cascas e/ou sementes e em pequenas porções individuais (Alves et al., 2000).

Os produtos minimamente processados (PMP) apresentam maior atividade metabólica, com elevada taxa respiratória e de deterioração, o que diminui relativamente a sua vida de prateleira. Logo, técnicas adequadas de conservação devem ser adotadas no sentido de se estender sua vida útil, preservando-se sua qualidade.

A utilização de atmosfera modificada vem apresentando bons resultados no armazenamento de PMP sob refrigeração, podendo ser obtida ativa ou passivamente. Na atmosfera modificada passiva, o produto é acondicionado em embalagem, e a atmosfera é modificada pela própria respiração do produto, em função da permeabilidade da embalagem e da temperatura. A atmosfera modificada ativa é criada injetando-se, inicialmente, no espaço livre da embalagem uma mistura gasosa predeterminada, sendo a atmosfera de equilíbrio determinada também pela interação entre o produto, embalagem e ambiente (Arruda, 2002).

Poucos estudos têm sido desenvolvidos em relação ao monitoramento dos níveis dos gases em embalagens de melões minimamente processados. Um dos maiores inconvenientes encontrados na utilização da atmosfera modificada é o acúmulo de metabólitos anaeróbicos, tais como acetaldeído e etanol no interior das embalagens, produzindo sabor e odor indesejáveis (Pesis et al., 2002).

$\mathrm{O}$ objetivo do presente trabalho foi avaliar as mudanças nas variáveis $\mathrm{pH}$, acidez titulável, sólidos solúveis, acetaldeído e etanol, associadas à qualidade de melões minimamente processados armazenados sob refrigeração e atmosfera modificada (passiva e ativas:
$5 \% \mathrm{O}_{2}+5 \% \mathrm{CO}_{2}$ e $2 \% \mathrm{O}_{2}+10 \% \mathrm{CO}_{2}$ ), e o acúmulo de $\mathrm{CO}_{2}$ no interior das embalagens.

\section{MATERIAL E MÉTODOS}

Melões 'Orange Flesh' provenientes de Mossoró - RN, foram adquiridos no comércio local de Lavras - MG, e transportados ao Laboratório de Bioquímica de Frutos (Pós-Colheita) do Departamento de Ciência dos Alimentos da Universidade Federal de Lavras.

Os melões foram lavados com detergente neutro e enxaguados em água corrente. Em seguida, foram imersos em solução de hipoclorito de sódio 200mg.L $\mathrm{L}^{-1}$ por 15 minutos. O processamento mínimo foi realizado em condições higiênicas, em que todos os utensílios foram previamente lavados e sanificados com soluções de hipoclorito de sódio $300 \mathrm{mg} . \mathrm{L}^{-1}$. Foram utilizados luvas, gorros, máscaras e aventais.

Os melões foram descascados manualmente, cortados ao meio, e as sementes retiradas; cada metade foi cortada em quatro fatias longitudinais e processadas em forma de leque com aproximadamente 2 $\mathrm{cm}$ de espessura, usando o multiprocessador MASTER AT. Em seguida, os leques foram imersos em solução de hipoclorito de sódio $100 \mathrm{mg} . \mathrm{L}^{-1}$ por 3 minutos e drenados com auxílio de peneira plástica. Os leques foram colocados em embalagens de polipropileno $(15,0 \mathrm{~cm}$ comprimento x $11,5 \mathrm{~cm}$ largura $x 4,5 \mathrm{~cm}$ altura), sendo estas seladas com filme flexível de polietileno + polipropileno $60 \mu \mathrm{m}$ de espessura, usando Seladora de Bandejas - AP340 (TecMaq) com a injeção inicial de duas misturas de gases, sendo $5 \% \mathrm{O}_{2}+5 \% \mathrm{CO}_{2}$ (atmosfera modificada ativa 1, AM1) e $2 \%$ $\mathrm{O}_{2}+10 \% \mathrm{CO}_{2}$ (atmosfera modificada ativa 2, AM2). As embalagens que foram apenas seladas sem a injeção de gases foram consideradas controle (atmosfera modificada passiva, AMP). Em seguida, foram armazenadas em câmara refrigerada $\left(6 \pm 1{ }^{\circ} \mathrm{Ce} 90 \pm 5 \%\right.$ UR) durante 8 dias. As seguintes análises foram realizadas a cada dois dias:

pH - utilizou-se pHmetro B474 da Micronal, segundo a técnica da AOAC (1992).

Acidez titulável (AT) - obtida por titulação com solução de $\mathrm{NaOH} 0,1 \mathrm{~N}$, tendo como indicador fenolftaleína, segundo a técnica

\footnotetext{
${ }^{1}$ (Trabalho 090/2004). Recebido: 14/07/2004. Aceito para publicação: 18/12/2004.

${ }^{2}$ Engenheira Agrônoma, Doutoranda em Ciências dos Alimentos, Departamento de Ciências dos Alimentos - DCA, Universidade Federal de Lavras - UFLA, LavrasMG, bmvboas@hotmail.com.

${ }^{3}$ Engenhera Agrícola, dra. Em Engenharia de Alimentos, Pesquisadora CNPq, DCA-UFLA, mônica@ufla.br.

${ }^{4}$ Engenheiro Agrônomo, Prof. Dr. Em Ciência dos Alimentos, DCA-UFLA, bolsista produtividade CNPq.

${ }^{5}$ Farmacêutica-Bioquímica, Doutora em Ciência dos Alimentos, DCA-UFLA.

${ }^{6}$ Bióloga, Prof. Dra. Em Ciência dos Alimentos, UERN, Mossoró-RN.
} 
estabelecida pelo Instituto Adolfo Lutz (1985). Os resultados foram expressos em \% de ácido cítrico.

Sólidos solúveis (SS) - determinados por refratometria, segundo a AOAC (1992), usando refratômetro digital ATAGO PR-100 com compensação de temperatura automática a $25^{\circ} \mathrm{C}$, e os resultados expressos em ${ }^{\circ}$ Brix.

Concentração de $\mathrm{CO}_{2}$ - Amostras gasosas foram retiradas do interior das embalagens utilizando seringas "gastight" $(100 \mu \mathrm{L}) \mathrm{e}$ injetadas em cromatógrafo a gás (Varian 3800), conectado a uma "workstation" (Varian Star 4.5) e equipado com detector de condutividade térmica e coluna Chromopak, utilizando como gás de arraste o nitrogênio, a um fluxo de $2,5 \mathrm{~mL} \cdot \mathrm{min}^{-1}$. A temperatura do forno foi de $220^{\circ} \mathrm{C}$. O volume injetado foi de $100 \mu \mathrm{L}$ e o resultado expresso em $\%$.

Acetaldeído e etanol - Num tubo de ensaio hermético com tampa de rosca e perfuração no centro, vedada por um septo, foram acrescentados: $2,5 \mathrm{~g}$ de polpa de melão, $2,5 \mathrm{~mL}$ de água destilada deionizada, $2,5 \mathrm{~g}$ de cloreto de sódio e $1 \mathrm{~mL}$ de butanol 50ppm. Levou-se à temperatura de congelamento de $-80^{\circ} \mathrm{C}$. Depois de congelados, os tubos contendo as amostras foram colocados em um bloco aquecedor, aumentando-se a temperatura gradativamente até atingir $80^{\circ} \mathrm{C}$. As amostras permaneceram nesta temperatura durante 1 hora, sendo homogeneizadas de 15 em 15 minutos com homogeneizador de tubos tipo Vortex. Após a homogeneização das amostras, coletaram-se $500 \mu \mathrm{L}$ do "headspace" através do septo, utilizando-se de uma seringa tipo "gastight", com posterior injeção em cromatógrafo. As análises de etanol e acetaldeído foram realizadas pelo método adaptado de Ott et al. (1997) e Abreu (1993), utilizando-se de um cromatógrafo a gás (Varian 3800), conectado a uma "workstation" (Varian Star 4.5). Utilizaram-se coluna cromatográfica Varian-Wax $(30 \mathrm{~m} \times 0,32 \mathrm{~mm} ; 0,25 \mu \mathrm{m})$ e detector de ionização de chama.

O experimento foi conduzido em delineamento inteiramente casualizado, com 3 repetições, em esquema fatorial 3 x 5, constituído pelos fatores atmosfera modificada (AMP, AM1 e AM2) e tempos de armazenamento $(0 ; 2 ; 4 ; 6$ e 8 dias). A parcela experimental foi constituída por uma embalagem contendo aproximadamente $190 \mathrm{~g}$ de melão minimamente processado. As análises estatísticas foram realizadas pelo programa SISVAR (Ferreira, 2000). Os dados foram submetidos à análise de variância, e as médias comparadas pelo teste de Tukey, a 5\% de probabilidade.

\section{RESULTADOS E DISCUSSÃO}

Houve interação significativa entre os fatores atmosfera modificada e tempos de armazenamento para a variável $\mathrm{pH}$. Os frutoscontrole (atmosfera modificada passiva) apresentaram $\mathrm{pH}$ superior aos armazenados sob atmosfera modificada ativa no segundo dia e superior aos acondicionados sob atmosfera modificada ativa $2 \% \mathrm{O}_{2}+10 \% \mathrm{CO}_{2}$ (AM2) no oitavo dia (Tabela 1). Os valores de $\mathrm{pH}$ variaram de 6,0 a 6,47, estando próximos aos encontrados por Pinto (2002), que também trabalhou com melões 'Orange Flesh' minimamente processados.

Os teores de acidez titulável (AT) oscilaram entre 0,043 e 0,096\% durante o período analisado (Tabela 1). Com exceção do segundo dia de armazenamento, quando os frutos-controle apresentaram valores de AT inferiores àqueles sob atmosfera modificada ativa, os frutos sob os três tipos de atmosferas não apresentaram diferenças significativas entre si. Pinto (2002) observou pequenas variações na AT de melões 'Orange Flesh' minimamente processados armazenados a 3 e $6^{\circ} \mathrm{C}$. Menezes (1996) afirma que o teor de ácidos orgânicos apresenta pouca contribuição para o sabor e aroma do melão, uma vez que a variação nos níveis de AT durante sua maturação tem pouco significado prático em função da baixa concentração dos mesmos.

Houve interação significativa entre os fatores atmosfera modificada e tempos de armazenamento para a variável sólidos solúveis (SS). Apenas no sexto dia de armazenamento foram observadas diferenças estatísticas, quando o controle apresentou maiores valores de SS (Tabela 2). Bai et al. (2001), trabalhando com melões 'Cantaloupe' minimamente processados, encontraram teores de SS em torno de 9,5\%, sendo que nenhuma mudança foi observada durante 12 dias de armazenamento, em todas atmosferas modificadas. Estes mesmos autores relataram que os valores de SS foram menores que os esperados, provavelmente devido à diluição provocada pelo banho de imersão em solução sanificante.

TABELA 2 - Valores médios de sólidos solúveis ( ${ }^{\circ}$ Brix) de melão 'Orange Flesh' minimamente processado armazenado sob atmosfera modificada passiva (AMP) e ativa (AM1 - 5\% $\mathrm{O}_{2}+5 \%$ $\left.\mathrm{CO}_{2} ; \mathrm{AM} 2-2 \% \mathrm{O}_{2}+10 \% \mathrm{CO}_{2}\right)$ e refrigerado $\left(6 \pm 1^{\circ} \mathrm{C}\right.$ e 90 $\pm 5 \%$ UR), por 8 dias.

\begin{tabular}{lccccc}
\hline Atmosfera & \multicolumn{5}{c}{ Tempos de armazenamento (dias) } \\
\cline { 2 - 6 } modificada & 0 & 2 & 4 & 6 & 8 \\
\hline AMP & $9,2 \mathrm{a}$ & $9,1 \mathrm{a}$ & $8,9 \mathrm{a}$ & $9,6 \mathrm{a}$ & $9,0 \mathrm{a}$ \\
AM1 & $9,5 \mathrm{a}$ & $8,5 \mathrm{a}$ & $8,9 \mathrm{a}$ & $8,4 \mathrm{~b}$ & $8,4 \mathrm{a}$ \\
AM2 & $9,2 \mathrm{a}$ & $8,5 \mathrm{a}$ & $8,4 \mathrm{a}$ & $8,1 \mathrm{~b}$ & $8,6 \mathrm{a}$ \\
\hline
\end{tabular}

${ }^{1}$ Médias seguidas de mesma letra na coluna não diferem entre si, pelo teste de Tukey, a 5\%.

Observou-se tendência normal a um acúmulo na concentração de dióxido de carbono $\left(\mathrm{CO}_{2}\right)$ no interior das embalagens devido ao processo de respiração do fruto (Figura 1). Os frutos sob atmosfera modificada passiva apresentaram, no interior da embalagem, aumento linear na concentração de $\mathrm{CO}_{2}$ durante todo o período analisado. $\mathrm{O}$ valor máximo, observado no oitavo dia, foi de aproximadamente $16 \%$. A concentração de $\mathrm{CO}_{2}$ aumentou de $5 \%$ a 23,45\%, nas embalagens com atmosfera modificada ativa $5 \%$ de $\mathrm{O}_{2}+5 \%$ de $\mathrm{CO}_{2}$ (AM1) até o $8^{\circ}$ dia de armazenamento. AAM2 permitiu a manutenção dos níveis de $\mathrm{CO}_{2}(10$ a $11,98 \%$ ) até o quarto dia de armazenamento, determinando um aumento mais acentuado do quarto ao sexto dia (11,98 a 20,21\%), com retorno à estabilidade até o $8^{\circ}$ dia $(21,86 \%)$, o que demonstra certa eficiência da AM2 no controle da atividade respiratória do melão minimamente processado até o $4^{\circ}$ dia. Os valores de etanol observados nas amostras acondicionadas sob $2 \%$ de $\mathrm{O}_{2}$ e $10 \%$ de $\mathrm{CO}_{2}$ sugerem que tal condição atmosférica possa ter induzido a respiração anaeróbica do produto a partir do quarto dia (Tabela 4). Bonnas (2002) também detectou aumento

TABELA 1 - Valores médios de pH e acidez titulável (\% ácido cítrico) de melão 'Orange Flesh' minimamente processado armazenado sob atmosfera modificada passiva (AMP) e ativa (AM1 - $\left.5 \% \mathrm{O}_{2}+5 \% \mathrm{CO}_{2} ; \mathrm{AM} 2-2 \% \mathrm{O}_{2}+10 \% \mathrm{CO}_{2}\right)$ e refrigerado $\left(6 \pm 1{ }^{\circ} \mathrm{Ce} 90 \pm 5 \%\right.$ UR), por 8 dias.

\begin{tabular}{|c|c|c|c|c|c|}
\hline \multirow{2}{*}{$\begin{array}{l}\text { Atmosfera } \\
\text { modificada }\end{array}$} & \multicolumn{5}{|c|}{ Tempos de armazenamento (dias) } \\
\hline & 0 & 2 & 4 & 6 & 8 \\
\hline \multicolumn{6}{|c|}{$\mathrm{pH}$} \\
\hline AMP & $6,07 \mathrm{a}$ & $6,43 \mathrm{a}$ & $6,23 \mathrm{a}$ & $6,13 \mathrm{a}$ & $6,47 \mathrm{a}$ \\
\hline AM1 & $6,07 \mathrm{a}$ & $6,13 b$ & $6,27 \mathrm{a}$ & $6,13 \mathrm{a}$ & $6,30 \mathrm{a}$ \\
\hline AM2 & $6,00 \mathrm{a}$ & $6,13 b$ & $6,10 \mathrm{a}$ & $6,10 \mathrm{a}$ & $6,03 \mathrm{~b}$ \\
\hline \multicolumn{6}{|c|}{ Acidez titulável } \\
\hline AMP & $0,096 \mathrm{a}$ & $0,043 \mathrm{~b}$ & $0,055 \mathrm{a}$ & $0,068 \mathrm{a}$ & $0,064 \mathrm{a}$ \\
\hline AM1 & $0,092 \mathrm{a}$ & $0,077 \mathrm{a}$ & $0,055 \mathrm{a}$ & $0,072 \mathrm{a}$ & $0,068 \mathrm{a}$ \\
\hline AM2 & $0,094 \mathrm{a}$ & $0,072 \mathrm{a}$ & $0,052 \mathrm{a}$ & $0,068 \mathrm{a}$ & $0,068 \mathrm{a}$ \\
\hline
\end{tabular}

${ }^{1}$ Médias seguidas de mesma letra na coluna não diferem entre si, pelo teste de Tukey, a 5\%. 
nos níveis de $\mathrm{CO}_{2}$ em abacaxi minimamente processado armazenado por 8 dias, a $5^{\circ} \mathrm{C}$, nas mesmas condições atmosféricas utilizadas neste trabalho. Os resultados encontrados estão de acordo com o previsto, pois quanto menor a quantidade de oxigênio fornecida, menor a taxa de respiração de um produto vegetal. O mesmo comportamento para esta variável foi encontrado por Freire Júnior (1999) trabalhando com alface minimamente processada armazenada em atmosfera modificada. Sabe-se que baixos níveis de $\mathrm{O}_{2}$ e altos níveis de $\mathrm{CO}_{2}$ têm efeito positivo na manutenção de frutas e hortaliças, reduzindo sua taxa respiratória. Entretanto, o mínimo de oxigênio entre 1 e 3\%, dependendo do produto, é requerido para impedir a respiração anaeróbica.

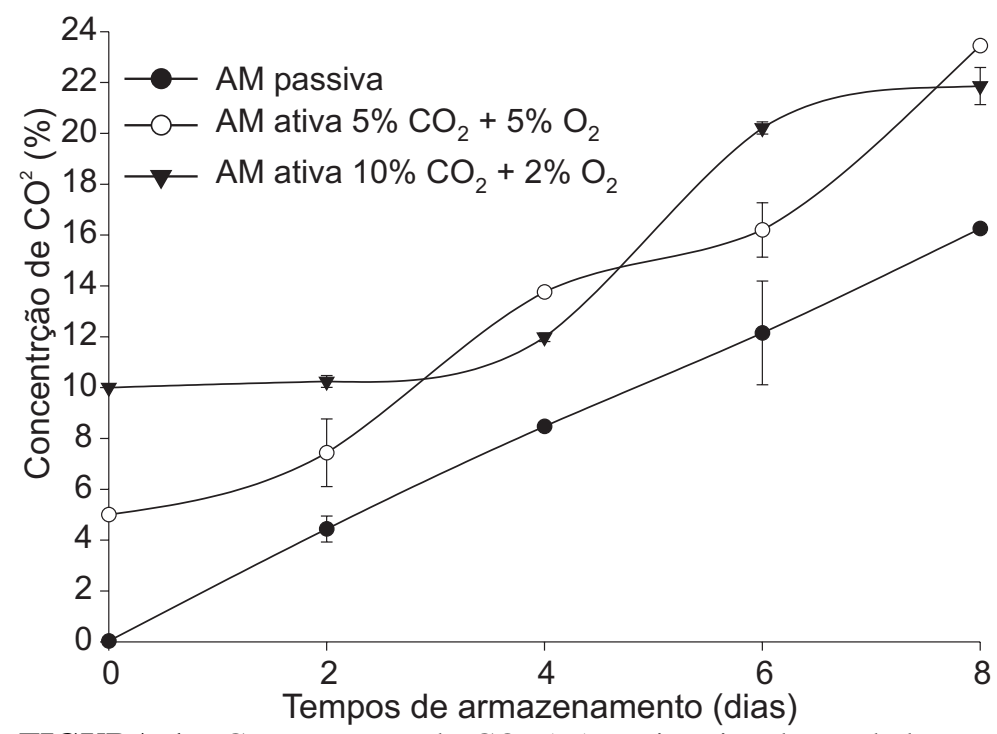

FIGURA 1 - Concentração de $\mathrm{CO}_{2}(\%)$ no interior das embalagens contendo melão 'Orange Flesh' minimamente processado armazenado sob atmosfera modificada e refrigerado $(6 \pm$ $1^{\circ} \mathrm{Ce} 90 \pm 5 \%$ UR), por 8 dias.
Os teores de acetaldeído diminuíram no decorrer do armazenamento em todos os tratamentos. Aparentemente, os teores de acetaldeído não foram influenciados pelas atmosferas estudadas (Tabela 3). Sob as condições de atmosfera modificada, a via glicolítica pode substituir o ciclo de Krebs, como principal fonte de energia necessária para o tecido vegetal. Assim, o piruvato não é mais oxidado e, sim, descarboxilado para formar acetaldeído, $\mathrm{CO}_{2} \mathrm{e}$, finalmente, o etanol. Dessa forma, pode-se atribuir a redução nos níveis de acetaldeído no melão minimamente processado, por ser este um composto intermediário na síntese do etanol. Observa-se que a redução nos valores de acetaldeído foi concomitante à elevação no conteúdo de etanol, discutido a seguir.

Os teores de etanol aumentaram com o tempo para todos os tratamentos (Tabela 4). No entanto, podemos observar que os tratamentos com atmosfera modificada ativa apresentaram valores de etanol superiores ao controle (AMP), a partir do $4^{\circ}$ dia de armazenamento, e que a AM2 determinou maiores valores de etanol que a AM1, a partir do $6^{\circ}$ dia, demonstrando que, quando se restringe o fornecimento de $\mathrm{O}_{2}$, há o favorecimento da formação de etanol. Concentrações muito baixas de $\mathrm{O}_{2}$ e muito altas de $\mathrm{CO}_{2}$ atuam sinergisticamente, aumentando significativamente a produção de metabólitos fermentativos. Agar et al. (1999) encontraram concentrações mais altas de acetaldeído e etanol em fatias de kiwi armazenadas sob baixa concentração de $\mathrm{O}_{2}(1 ; 2$ e $4 \%)$ quando comparadas com aquelas armazenadas em ambiente com ar purificado (controle).

Bonnas (2002), trabalhando com abacaxi minimamente processado armazenado sob atmosfera modificada, também observou aumento nos teores de etanol em função do tempo, notadamente no sexto dia, porém, pela análise sensorial, não se detectou desenvolvimento de sabores e aromas desagradáveis no produto até o $8^{\circ}$ dia de armazenamento.Em melões minimamente processados, o desenvolvimento de sabores e aromas desagradáveis foi relacionado à presença de fungos na superfície dos cubos e não à respiração anaeróbica nos tecidos (Bai et al., 2001).

TABELA 3 - Valores médios de acetaldeído (ppm) de melão `Orange Flesh' minimamente processado armazenado sob atmosfera modificada passiva (AMP) e ativa (AM1 - 5\% $\left.\mathrm{O}_{2}+5 \% \mathrm{CO}_{2} ; \mathrm{AM} 2-2 \% \mathrm{O}_{2}+10 \% \mathrm{CO}_{2}\right)$ e refrigerado $\left(6 \pm 1^{\circ} \mathrm{Ce} 90 \pm 5 \% \mathrm{UR}\right)$, por 8 dias.

\begin{tabular}{|c|c|c|c|c|c|c|c|c|c|c|}
\hline \multirow{2}{*}{$\begin{array}{l}\text { Atmosfera } \\
\text { Modificada } \\
\end{array}$} & \multicolumn{10}{|c|}{ Tempos de armazenamento (dias) } \\
\hline & \multicolumn{2}{|c|}{0} & \multicolumn{2}{|c|}{2} & \multicolumn{2}{|c|}{4} & \multicolumn{2}{|c|}{6} & \multicolumn{2}{|c|}{8} \\
\hline & 0,5128 & 0,05 & 0,4962 & 0,02 & 0,4206 & 0,04 & 0,3519 & 0,08 & 0,2988 & 0,11 \\
\hline AM1 & 0,5244 & 0,09 & 0,4998 & 0,07 & 0,4601 & 0,08 & 0,4168 & 0,10 & 0,3929 & 0,07 \\
\hline AM2 & 0,5401 & 0,02 & 0,5100 & 0,06 & 0,4532 & 0,03 & 0,4155 & 0,04 & 0,3867 & 0,06 \\
\hline
\end{tabular}

TABELA 4 - Valores médios de etanol (ppm) de melão 'Orange Flesh' minimamente processado armazenado sob atmosfera modificada passiva (AMP) e ativa (AM1 - 5\% $\left.\mathrm{O}_{2}+5 \% \mathrm{CO}_{2} ; \mathrm{AM} 2-2 \% \mathrm{O}_{2}+10 \% \mathrm{CO}_{2}\right)$ e refrigerado $\left(6 \pm 1^{\circ} \mathrm{Ce} 90 \pm 5 \% \mathrm{UR}\right)$, por 8 dias.

\begin{tabular}{|c|c|c|c|c|c|c|c|c|c|c|}
\hline \multirow{3}{*}{$\begin{array}{l}\text { Atmosfera } \\
\text { Modificada } \\
\text { AMP }\end{array}$} & \multicolumn{10}{|c|}{ Tempos de armazenamento (dias) } \\
\hline & \multicolumn{2}{|c|}{0} & \multicolumn{2}{|c|}{2} & \multicolumn{2}{|c|}{4} & \multicolumn{2}{|c|}{6} & \multicolumn{2}{|c|}{8} \\
\hline & 0,7380 & 0,18 & 1,3033 & 0,17 & 1,6154 & 0,08 & 1,9861 & 0,08 & 2,3321 & 0,07 \\
\hline AM1 & 0,7334 & 0,13 & 1,3698 & 0,05 & 1,7721 & 0,06 & 2,1326 & 0,12 & 2,8948 & 0,08 \\
\hline AM2 & 0,7372 & 0,10 & 1,4964 & 0,06 & 1,8775 & 0,05 & 2,5678 & 0,04 & 3,1523 & 0,09 \\
\hline
\end{tabular}

\section{CONCLUSÕES}

1. A AM passiva proporcionada pelo acondicionamento do melão "Orange Flesh" minimamente processado em embalagem de polipropileno selada com filme flexível de polietileno + polipropileno $(60 \mu \mathrm{m})$, assim como as AM ativas $\left(5 \% \mathrm{O}_{2}+5 \% \mathrm{CO}_{2}\right.$ e $\left.2 \% \mathrm{O}_{2}+10 \% \mathrm{CO}_{2}\right)$ não interferem no $\mathrm{pH}, \mathrm{AT}, \mathrm{SS}$ e acetaldeído durante 8 dias sob condições de $6 \pm 1^{\circ} \mathrm{Ce} 90 \pm 5 \%$ UR.

2. A atmosfera modificada ativa $\left(2 \% \mathrm{O}_{2}+10 \% \mathrm{CO}_{2}\right)$ é mais eficaz no controle da atividade respiratória somente até o $4^{\circ}$ dia de armazenamento a $6 \pm 1^{\circ} \mathrm{C}$ e $90 \pm 5 \%$ UR.

\section{REFERÊNCIAS}

ABREU, L. R. Factors affecting the biossyntesis of branched-chain fatty acids in milk fat. 1993. 163f. (Doctorate Thesis in Food Science) University of Wisconsin, Madison.

AGAR, I. T.; MASSANTINI,R.; HESS-PIERCE, B.; KADER, A. A. Postharvest $\mathrm{CO}_{2}$ and ethylene production and quality maintenance of fresh-cut kiwifruit slices. Journal of Food Science, Chicago, v.64, n.3, p. 433-440, 1999.

ALVES, R. E.; SOUZA FILHO, M. de S. M. de; BASTOS, M. do S. R.; FILGUEIRAS, H. A. C.; BORGES, M. de F. Pesquisa em 
processamento de frutas no Brasil. In: ENCONTRO NACIONAL SOBRE PROCESSAMENTO MÍNIMO DE FRUTAS E HORTALIÇAS, 2., 2000, Viçosa. Palestras... Viçosa: UFV, 2000. p. 75-85.

ARRUDA, M. C. de. Processamento mínimo de melão rendilhado: tipo de corte, temperatura de armazenamento e atmosfera modificada. 2002. 71f. Dissertação (Mestrado em Agronomia)-Escola Superior de Agricultura Luiz de Queiroz, Universidade de São Paulo, Piracicaba, 2002.

ASSOCIATION OF OFFICIALANALYTICAL CHEMISTRY. Official methods of analysis of the Association of Official Analytical Chemistry. 12.ed. Washington, 1992. 1.015p.

BAI, J. H.; SAFTNER, R. A.; WATADA, A. E.; LEE, Y. S. Modified atmosphere maintains quality of fresh-cut cantaloupe (Cucumis melo L.). Journal of Food Science, Chicago v.66, n.8, p. 1207-1211, 2001.

BONNAS, D. S. Qualidade do abacaxi cv. Smooth cayenne minimamente processado embalado sob atmosfera modificada. 2002. 100f. Tese (Doutorado em Ciência dos Alimentos) - Universidade Federal de Lavras, Lavras-MG, 2002.

FERREIRA, D. F. Análises estatísticas por meio do SISVAR para windows versão 4.0. In: REUNIÃO ANUALDA REGIÃO BRASILEIRADA SOCIEDADE INTERNACIONAL DE BIOMETRIA, 45., 2000, São Carlos. Programa e Resumo...São Carlos: UFSCar, 2000. p.235.
FREIRE JUNIOR, M. Efeito da temperatura de armazenamento e da atmosfera modificada na qualidade da alface hidropônica cv. Regina minimamente processada. 1999. 120f. Tese (Doutorado em Ciência dos Alimentos) - Universidade Federal de Lavras, Lavras-MG, 1999.

INSTITUTO ADOLFO LUTZ. Normas analíticas, métodos químicos e físicos para análise de alimentos. 3.ed. São Paulo: Instituto Adolfo Lutz, 1985. v. 1, 533p.

MENEZES, J. B. Qualidade pós-colheita de melão tipo Gália durante a maturação e o armazenamento. 1996. 157f. Tese (Doutorado em Ciência dos Alimentos) - Universidade Federal de Lavras, Lavras/ MG, 1996.

OTT, A.; FAY, L. B.; CHAINTREAU, A. Determination and origin of the aroma impact compounds of yogurt flavor. Journal of Agricultural and Food Chemistry, Easton, v.45, n.3, p. 850-857, 1997.

PESIS, E.; DVIR, O.; REYGENBERG, O.; ARIC, R. B.;ACKERMAN, M.; LICHTER, A. Production of acetaldehyde and ethanol during maturation and modified atmosphere storage of litchi fruit. Postharvest Biology and Technology, Boca Raton, v.26, p.157-165, 2002.

PINTO, S. A. A. Processamento mínimo de melão tipo Orange Flesh e de melancia 'Crimson Sweet'. 2002. 120f. Dissertação (Mestrado em Agronomia/Produção Vegetal) - Universidade Estadual Paulista, Faculdade de Ciências Agrárias e Veterinárias, Jaboticabal-SP, 2002. 